\title{
The Relevance of Blockchain for Collaborative Networked Organizations
}

\author{
Hans Schaffers \\ Adventure Research, 7231GL Warnsveld, The Netherlands \\ hsladventureresearch.nl
}

\begin{abstract}
Blockchain, a distributed secure digital ledger technology, is a relatively recent development with potentially transformational implications for economy and society. Its specific characteristics enable new decentralized models of distributed and trusted transactions. This position paper explores the implications of blockchain for collaborative networked organizations. In particular we aim at understanding the implications for companies in various economic sectors, and how new forms of networked organizations and new business models will be enabled. We also will focus on enablers of blockchain innovations, in particular with respect to governance of blockchain-based platforms and business networks. The paper results in a discussion of research challenges in the field of blockchainenabled collaborative networked organizations.
\end{abstract}

Keywords: Blockchain, Organizations, Collaboration, Networks, Governance.

\section{Introduction}

Over the last years, blockchain technology and its applications received a lot of attention in business, academia and society at large [1]. According to a recent survey, $77 \%$ of FinTech companies expect to adopt blockchain as part of a production system or process by 2020 [2]. A lot of innovation is going on, mostly in the domain of financial services and payments systems, however use case experimentation and piloting is proceeding in many other sectors including logistics and supply chains, education, healthcare, government services and energy.

Key players in the blockchain ecosystem are diverse [3]. Blockchain platforms such as Ethereum, Hyperledger, NEO, Coinbase, the R3 consortium and Ripple enable developers to develop smart contract and distributed applications. Large financial institutions are involved, including large banking and insurance companies, as well as accountancy firms, venture capitalists, and computing firms such as IBM who offer blockchain platform and enterprise solutions. Increasingly there is interest from the side of national and global regulators, governments and standardization organisations.

Blockchain technologies and current developments are explained in many publications such as [1], [3], [4], [5]. In addition, there exist a variety of lively blogs discussing blockchain technological developments, use cases, governance issues and more. Blockchain technology is based on distributed digital ledger technology, recording cryptographic secured transactions in chains of chronologically connected, stored and 
timestamped blocks. As such blockchain is based on a new form of distributed consensus of transactions and in principle enables peer-to-peer economies. Its importance goes far beyond its most prominent application, the cryptocurrency called Bitcoin. Blockchain has the wide-ranging potential to enable radically new decentralized forms of organization and radically new business models.

Based on a review of literature, this paper aims to explore, examine and discuss how blockchain affects forms of collaborative networked organisations and to understand the determinants of adoption and transition towards blockchain economies. The precise impacts of blockchain on economy and society are still unclear. Internationally a range of blockchain pilots are being carried out to understand these impacts as well as their business models, governance frameworks and other key factors for adoption, scaling up and success. Section 2 presents an overview of blockchain technology and impacts.

At the intersection of blockchain technology and organizational change several challenging issues come together. Section 3 therefore aims to understand the underlying conditions for blockchain-enabled forms of collaborative organizations. Section 4 takes a closer look at the relevance of blockchain for collaborative networked organizations and how these are enabling new ways of working and doing business. A central adoption issue, addressed in section 5, is the governance and decision-making mechanisms in blockchain-enabled decentralized organizations. Finally, challenges and research opportunities for the future are being discussed in section 6 .

\section{Blockchain Technology and Impacts}

\subsection{Blockchain as Technological Innovation}

The origin of blockchain technology lies in a protocol for peer-to-peer transactions developed by Satoshi Nakamoto, a pseudonym [4]. Nakamoto describes the digital currency bitcoin as 'peer-to-peer electronic cash system, allowing online payments to be sent directly from one party to another without going through a financial institution'. The underlying blockchain technology is based on applying peer-to-peer distributed timestamps to generate computational proof of the chronological order of transactions. As [4] explains, blockchain is a collection of blocks which are chronologically linked and cryptographically secured, and its data are stored immutable in a shared database, the digital ledger. The software system enables the validation of transactions by consensus mechanisms within the network of all participants. This way blockchain technology allows parties to engage in transactions directly, whereas the integrity of the data involved in the transactions is ensured without the need for a trusted third party. All participants to a blockchain have access to and can store and modify a copy.

A key innovation for blockchain has been the possibility of smart contracts, which are based on software rules for execution of blockchain transactions, contingent on events or conditions, as explained in [6] and [7]. Such smart contracts can be stored as transactions in the distributed ledger. This principle has a wide range of application possibilities varying from automated execution of contingent claims (insurance and finance [8]) to, in the future, industrial applications in the scope of Internet of Things and big data. Smart contract tools are offered for example by the Ethereum and Hyperledger Fabric platforms. A related development pursued by Ethereum is that a set of smart 
contracts creates a so-called Decentralized Autonomous Organization [9]. An example is The DAO crowdfunding platform created in 2016 upon the Ethereum blockchain, functioning as investment fund for start-ups where funding comes from the Ether cryptocurrency functioning as shares. The DAO subsequently experienced an infamous hack, which reminds us of the complexities of such systems and of the vulnerabilities in using the in itself extremely secure blockchain and smart contracts technology.

\subsection{Blockchain Platforms}

Blockchain applications normally require collaboration among parties involved e.g. for trading and supply chain management. Blockchain applications can be implemented through existing blockchain platforms; alternatively, parties can decide to build and operate their own blockchain system [10]. The different options available are connected to the operating models and governance frameworks of existing blockchain platforms [11]. In addition, these choices are dependent on the business model and trade-offs in terms of control and ownership on the side of the application owner (user).

In this context, the distinction between private and public blockchain platforms is essential and a lot of debate is going on regarding this issue [12]. A public ('permissionless') blockchain platform such as Bitcoin and Ethereum is completely open in terms of participation and access agreements. However, Ethereum is also an infrastructure which enables building distributed organizations based on smart contracts [8]. A private ('permissioned') blockchain builds on permission for modifying the ledger and access agreements. Examples of permissioned blockchains are Ripple and Hyperledger Fabric (hosted by The Linux Foundation).

A specific form of private blockchain is consortium blockchain, where participants for a consortium which is governed by consortium agreements. For example, IBM Blockchain Platform, making use of Hyperledger technologies, is a service for companies to help creating, operating and governing their private blockchain applications. Setting up private consortium blockchains implies a variety of important issues, such as the process of consortium building, partner selection, consortium composition and extension, dispute settling, rules for network governance, and legal and regulatory aspects. There is an interesting relationship with the field of collaborative networked organizations where comparable type of issues are in discussion [13].

\subsection{Blockchain Applications and Business Models}

As such, blockchain goes far beyond cryptocurrency bitcoin payments as it records and keeps track of all kinds of transactions and mutations. Think of use cases in financial transactions, insurance claim process, transfer of all kinds of asset ownership, and making changes in complex contracts and records. Given its emphasis on value ownership and exchange, blockchain is sometimes called the 'Internet of Value' [1].

The first and most prominent application of blockchain technology has been the Bitcoin blockchain, which has a wide range of implications for the economy and financial system as explained in [14] and [15]. The precise reasons why bitcoin as digital currency system represents value are analysed in [16], although there is definitely some controversy in this matter. However, the underlying blockchain technology is of wider 
relevance for the economy [17] as it provides a platform for decentralized secure economic transactions in general, affecting the financial industry, insurance, supply chain management, energy markets, healthcare and many more. Interestingly, blockchain applications are often crossing the borders of sectors and probably it is there where the most attractive and innovative use cases lie. Some examples of potential blockchain applications discussed in literature are the following:

- Financial transactions. New financial instruments such as micro-payments, peer-topeer lending, trading records and smart contracts can be built upon blockchain [18].

- Business process management. Applications of blockchain technology for business process management are described in [19], demonstrating its application potential in interorganizational transaction settings and discussing governance and accountability aspects and how smart contracts may provide solutions. In [20], a prototype blockchain application for cross-organizational workflow management in a financial institution is presented applied to document workflow for letter of credit. The prototype was implemented on a private Ethereum blockchain enabling smart contracts. The paper concludes that blockchain increases auditability, trust, efficiency and improves workflow management. However, remaining challenges include process and document standardization and regulation.

- Insurance. Given the event-driven nature of insurance use cases, blockchain and smart contracts may have considerable implications. Examples presented in [8] include claim processing and automatic refund, crop insurance in relation to weather data, identity verification and fraud prevention, and pay-per-use insurances.

- Auditing. Blockchain technology provides much opportunity for auditing. [21] discusses automated audits based on correctness analysis of statements. Given the opportunities for fraud in the software environment the paper states that security of the underlying environment and IT systems controls is critical for adoption.

- Education. A Joint Research Centre study provides an overview of blockchain education opportunities [22]. Examples are the issuing of certificates, storing verified eportfolios, and managing intellectual property rights. An interesting use case is storage of qualifications on a blockchain. An example, elaborated in the report, is how the Open University in the UK is working on standards for badging, certification and reputation with the use of blockchain as a trusted ledger. The report also states that educational business models might very well change in using blockchain technology, as administration costs will decrease and fraud reduced. In the future, students could benefit from increased flexibility in selecting courses á la carte.

- Supply chain management. Blockchain technology may increase transparency and traceability of supply chains. In [23], blockchain adoption bottlenecks are addressed both on the supplier and consumer sides. In [24] a case study of supply chain integration based on blockchain is presented, concluding that functionalities such as timestamps and smart contracts were beneficial. The paper also identifies a need for standards and interoperability, which blockchain itself does not offer.

- Assets sharing. Blockchain technology combined with Internet of Things creates interesting application opportunities in the sharing economy [25]. Blockchain could avoid dominance of dominant platform players such as Airbnb and Uber [3]. 
- Real estate transactions. Many intermediary parties are involved, and blockchain is supposed to transform the role of intermediaries such as notaries and cadastre. These actors are looking for new roles and services in the blockchain-enabled value chain.

- Open innovation, co-creation and IP management. Blockchain could support the tracking, management and exchange of intellectual property rights. This has also been an issue since long in the Collaborative Networked Organizations community.

Of high interest is the potential of blockchain within the domain of Industry 4.0. Industry 4.0 focuses on applying the concept of Internet of Things within the smart factory and emphasizes how smart factories are connected within a wider business ecosystem [26]. Different layers of applying smart technologies can be distinguished: the smart plant built around autonomously operating and interconnected cyber-physical systems, the smart factory enabling responsive and adaptive manufacturing automation and the smart business network or ecosystem enabling demand-driven flexible supply chain management. Industry 4.0 enables network-centric production methods, smart product and service concepts and responsive business models in collaborative ecosystems.

Blockchain and smart contracts fits well into the network-centric Industry 4.0 paradigm. Applications to supply chain management, product tracking and auditing are currently being worked on. [27] describes a number of applications, including a pilot in which IBM and Maersk tested the application of blockchain in logistics and supply chain management focusing on the tracking of containers during shipping movements. Foreseen are also applications of blockchain smart contracts in combination with data analytics and smart sensor systems (Internet of Things). The paper also identifies technical and organizational issues to be resolved, such as scalability and latency, lack of standards, and establishing the appropriate legal and regulatory conditions.

Probably the most interesting applications of blockchain in Industry 4.0 will be found in logistics, asset management and supply chain management. A particular interesting possibility could be how blockchain technology could support the response to unexpected disturbances within the supply chain. Given the lack of satisfactory interoperability among enterprise systems manual interventions are often required. Application of blockchain and smart contracts technology could facilitate rescheduling, replanning and contracting, making supply chains more resilient, responsive and adaptive.

Adoption of blockchain technologies will have profound impacts on the company business model, including the structure of partnerships, the corporate governance model, the financial model and the technical infrastructure and there is a growing literature in this field. For example [28] examines the different ways how key elements of corporate business models are affected. For example, the support of micropayments and the increased transaction security alters the revenue model, simplifies delivery processes and facilitates extension of the business network.

As a conclusion so far, blockchain will not only affect the business models of individual corporations but will require modified business models at the level of collaborative networks. Blockchain changes the coordination of economic activities in business networks, and blockchain applications require new forms of partnerships and collaboration networks established in consortia or other forms of networked organization. This also implies the need to establish appropriate governance models not only at the level of individual organizations but at the level of collaborative networks. 


\section{Blockchain and Economics of Governance}

\subsection{Blockchain as Governance}

We now turn to understanding the nature of blockchain-enabled organizations. Blockchain can be understood as one form of governance, the same way markets, hierarchies and networks are forms of governance suitable in different situational contexts. This perspective originates mostly from the influential paradigms of transaction cost economics [29] and theory of the firm [30], where organizations are seen as "nexus of contracts'. Williamson's organizational failures framework [29] looks at economics of organization from the perspective of contracts and transaction costs and analyses how both environmental factors (uncertainty and complexity, small numbers exchange relations) and human factors (bounded rationality and opportunism, also in relation to exploitation of trust) require 'governance'. Situationally different generic governance mechanisms can be identified with generic forms, markets, hierarchies and relational contracts. This has been explored not only in finance but also in organizational science studies in relation to network governance [31].

Of interest is the relation of these concepts to the characteristics of blockchain technology. In [32], building on transaction cost economics and public choice economy, blockchain is presented as alternative governance model, a specific type of decentralized organization. Given its transformational potential [32] considers blockchain not just as a new type of information technology but more institutional as a 'technology of governance' The paper argues that blockchain-enabled smart contracts and Decentralized Autonomous Organization will, through their transparency and decentralization characteristics, modify transaction costs. However, blockchain relates to complete contracts in the sense of anticipating to all potential future states, whereas in reality organizations address incomplete contracts characterized by uncertainties, so that most organizations are built upon governance arrangements to provide incentives and trust for cooperation. Clearly this implies that there are limits to blockchain-enabled decentralized autonomous organizations.

Interestingly [32] also addresses the role of collective decision-making rules and procedures. Blockchain is considered as 'trustless commons' where rules are embedded in smart contacts. This discussion bridges to the concept of peer production and sharing as developed in [33] and one direction for future innovation could be to understand the potential of blockchain for such commons-based models. [32] also refers to the importance of the governance of common pool resources as explored by Eline Ostrom, who proposed 'design rules' for successful commons self-governance. It can be expected that insight in how these governance-related issues are relevant for blockchain will be highly relevant for wider scale adoption of blockchain.

\subsection{Impact on Intermediaries}

Blockchain enables companies to decrease their transaction costs, with profound effects on the nature of companies: how they are funded and managed, how they create value and how they perform basic functions such as marketing, accounting, human resources, 
procurement, legal affairs [1]. Blockchain's technological characteristics enable extremely decentralized and self-organised forms of organization, aimed at creating, executing and managing transactions. Given the blockchain impact on transaction costs, blockchain-enabled organizational networks constitute an alternative with respective to traditional structures such as firms, supply chains and markets [34].

This may lead to disintermediation: intermediaries such as financial institutions and lawyers might no longer be needed in the future [32] and are exploring different roles and business models, as business networks based on blockchain could be better suited for creating products and services than traditional vertical integration [35]. For example, the role of intermediary platform organizations such as banks, insurance companies, auditors will be strongly affected, in particular through blockchain-enabled smart contracts, as blockchain enables bypassing such intermediaries through enabling peerto-peer transactional relations.

Whereas these intermediaries will be affected structurally, the business models of many other organizations, supply chains and business networks will be affected due to the mentioned new technical opportunities for trustless secure transactions enabled by blockchain technology. Also, traditional platform models where large intermediaries owning the platforms capture the vale may become affected by blockchain applications [17]. Blockchain-based applications such as the OpenBazaar marketplace are able to coordinate the common activities of a large number of individuals without the help of a third party, in a secure and decentralized manner [17]. New cooperative models are emerging where users are contributors as well as shareholders.

\section{Blockchain and Collaborative Networked Organizations}

\subsection{Collaborative Networked Organizations}

The previous section looked into the fundamentals of blockchain-enabled organizations. We now take a closer look to how blockchain relates to collaborative networked organizations. The latter concept focuses on collaboration for value creation across the boundaries of teams and organizations and within communities and associated forms of distributed collective intelligence. In [36], a collaborative network is defined as 'a network consisting of a variety of entities (e.g. organizations and people) that are largely autonomous, geographically distributed, and heterogeneous in terms of their operating environment, culture, social capital and goals, but that collaborate to better achieve common or compatible goals, thus jointly generating value, and whose interactions are supported by a computer network'. A diversity of forms exists; examples of types of collaborative networks are the professional virtual community, the extended enterprise, a business ecosystem, a virtual team, a virtual enterprise a supply chain. Key issues determining the success of collaborative networks are the building and maintaining of trust [37], the system of incentives, rules of fairness and sharing benefits, transparent governance principles [38], and alignment of values of participants [13].

Since long, issues such as trust, governance and culture have been important in connection with collaboration-oriented technological innovations. Over time, discussion 
has moved from technologies enabling collaborative working and e-business to technologies, business models and governance principles for business networks and more recently platform-based ecosystems [39].

\subsection{Blockchain-enabled Collaborative Organizations}

The issue is now how blockchain could contribute innovative elements to collaborative networked organizations, and how blockchain applications could benefit from collaborative networks organizations. This is an area which is open for new research, and the below aims to provide some initial views based on some available studies.

As regards trust and governance mechanisms, blockchain technology pursues trustless transaction environments. The fundamental approach of public, open blockchain technology developed by Nakamoto [4] is to enable secure peer-to-peer transactions, and in this perspective, 'blockchain is governance'. Still, blockchain has some security challenges as mentioned in section 2 . However, these vulnerabilities seem to be part of the technical and organizational environment of blockchain applications rather than to the blockchain and smart contracting technologies.

Within collaborative networked organizations research, mechanisms to build and maintain trust among participants and to create governance mechanisms have received attention [36], [38], [40]. Also, ownership and control issues as well as business models are important to address, otherwise partnering will fail. Consortium formation, definition of governance principles, partner selection and role definition are important activities in setting op and evolution of such networks. For example, [13] discusses the alignment of values of participants in the formation and evolution of collaborative networks. Therefore, an interesting research issue would be how tools for partner selection and other aspects of governance could be designed applicable in the lifecycle management of blockchain-enabled collaborative networks.

Trust, control and governance mechanisms remain particularly relevant at the level of private and consortium blockchains, where business networks and consortia are building and operating a blockchain application. Blockchain solutions require a collaboration among many different players in a viable ecosystem. Selection of participants and establishing a clear governance model is therefore important, already in the development phase [10]. Here comes in a number of the same issues as in collaborative networked organizations: participant selection, consortium sizing, joining and leaving procedures, consortium goals agreement, consensus building, liability, KPI's, platform management and decision making.

Blockchain could have implications not only for e-business transaction processes but also for human-oriented collaboration environments and virtual teams. Think of collaboration platforms which support distributed teams, such as shared workspace BSCW, having a wide range of functionalities such as document sharing, versioning, calendaring, presence, project management, conferencing and many more. Blockchain technology provides the opportunity to facilitate groupwork in terms of immutable projectrelated transactions to be registered on a blockchain. Here we see the distinction between blockchain as a formal procedural solution and the social trust-based system of group collaboration. 
The view of organizations as sets of transactional contracts has great value but also its limits. Organizations are complex socio-technical systems, where we see a variety of formal and informal, even spontaneous, structures and processes mediated through human interactions, team work, organizational procedures and rules, governance structures, leadership, and organizational culture. Organizations understood as nexus of contracts is a valuable perspective, focusing on transaction processes between parties that can be automated based on data such as orders, reservations and payments, on the other hand, the context of such transactions is human, social and cultural.

\section{Governance of Blockchain Ecosystems}

Governance is an issue to be considered in different contexts and at different levels of abstraction. Blockchain as a structural form of governance (as alternative for markets and hierarchies) was discussed in section 3. This should be distinguished from governance as a process, to be applied to blockchain applications, platforms and ecosystems and at the global level comparable with internet governance, as discussed in [1] and [3].

At a fundamental, global level [3] takes a point of departure in how governance of the internet is currently arranged (with entities such as ICANN, IETF, the WWW Consortium and other) in order to learn about implications for global blockchain governance. This level of governance agrees on general rules, is setting standards, agrees on and maintains policies, engages in knowledge development, implements watchdog functions, and builds out the global infrastructure. In [3] a self-organised multi-stakeholder governance structure for blockchain as a global resource - Internet of Value - is discussed, made up of various types of networks and institutions. The vision is that as a global resource, and from the need to protect consumers, citizens and companies in relation to the involved risks, blockchain requires global 'stewardship', which also includes legal frameworks and standardization.

Besides at the global ecosystem level, [1] envisions governance at the level of platforms and applications. At the level of blockchain platforms such as Ethereum, Ripple and Hyperledger, governance challenges include issues such as scaling, the creation of shared views within the blockchain community, the incentives for mass collaboration on innovations, how to agree on standards enabling interoperability, and how to address threats such as The DAO hack. At the level of blockchain applications, [1] proposes governance mechanisms in relation to assets and tools that run on platforms such as digital currencies and smart contracts, and applications such as payments, smart contracts, insurance which raise specific governance issues. Also, attention to platform and application interoperability will be important according to [1].

In this context, a debate is ongoing in relation to the need for blockchain control and governance. One of the controversies is whether governance should be implemented through blockchain code, or whether blockchain is part of a wider socio-technical system requiring governance and legislation. Given the apparent legal challenges and risks of blockchain-enabled transactions in the societal domain, such as liability, responsibility and enforceability, governance and regulatory challenges will require more attention [1]. This vision is also shared in [41], stating that blockchain technology operates independently from any centralized institutions or trusted authorities and implements their own internal system of rules, almost exclusively governed by the rules of code. 
This discussion actually raises the fundamental debate about autonomy and self-organization vs control and regulation, about whether individual freedoms are restrained rather than enhanced, and whether regulation constrains innovation opportunities, as raised in [32] and [42].

For example, [43] asserts that although blockchain governance is encoded in the blockchain protocols, it is still necessary to trust the functioning of the code as expected. We already referred to the hack of The DAO, where a vulnerability allowed for diverting a large amount of funds, while this did not violate Ethereum's of The DAO's rules or legal frameworks itself. In words of [46]: 'Code is law for machines, law is code for people'. A related view is [44], stating that when rules embedded in blockchain software could favour some companies at the expense of others, the authority to change the underlying rules becomes critically important. Governance mechanisms in order to agree on software changes, including dispute resolutions, sanctions, and enforcement of penalties will be needed. In a private or permissioned blockchain this could be similar to agreeing on a partnership agreement. In the same line of argument [45] states that the vision of 'Blockchain as governance' which indeed can lead to completely different ways of organizing economies has its limits: blockchains are part of a development and change process of a social nature, and thus are complex socio-technical systems. It is asserted that blockchains, by regulating human exchange, have serious distributional, ethical, and political consequences. Governance rules will be needed and this will raise difficult issues.

There is also ongoing work to resolve governance issues through technical mechanisms. In [46] an interesting blockchain-powered blueprint for a shared and public programmable economy is presented focusing on digital identities, blockchain-based trust, programmable money and marketplaces. Interestingly, some researchers working on blockchain applications for business process management are aware of governance issues. In [19] agency problems and incentives are found of high importance. The paper states that smart contracts could establish new governance models such as in decentralized Autonomous Organizations. However, in the light of our discussion it remains to be seen whether this approach is appropriate in terms of overcoming agency issues in business networks.

Governance in Blockchain platforms may learn from governance of network governance and governance of platform ecosystems in general. [39] discusses platform governance in terms of decision rights partitioning, control, and ownership, and identifies a central governance challenge in retaining sufficient control to ensure the platform integrity while relinquishing enough control to encourage innovation by developers. It would be an interesting topic for research to study the mechanisms of platform governance and governance in collaborative networked organisations and examine what is relevant for blockchain governance, given the situational context and the specific architectural and technological system embedded in the blockchain ecosystem.

\section{Challenges of Blockchain in Collaborative Environments}

Clear benefits of blockchain technology are the highly secure transactions made possible by blockchain technology. However, many literature sources point to challenges in terms of vulnerabilities, scale and performance, interoperability and standardization, 
new business models, governance mechanisms, legal and regulatory issues, and associated with all these issues, adoption, upscaling and acceptation of blockchain.

Interoperability and standards across different blockchain platforms and applications will be required. ITU, ISO and other organizations are working on standards terminology, reference architecture, identity management, security and many other topics. The Blockchain Interoperability Alliance has been set up in 2017 as a collaboration between parties aiming to connect various blockchain protocols and establish industry standards. Interoperability could help creating a richer ecosystem of and enable the creation of cross-blockchain smart contracts.

Now that blockchain technology has become more mature, also academic research has addressed various aspects of blockchain. Several publications have highlighted the new research issues associated with blockchain. For example, [47], in their introduction to a special issue of Business \& Information Systems Engineering, emphasizes the relevance of blockchain technology for information systems research. Potential challenging research areas are disruption of existing and creation of new business models; technical, economic and environmental sustainability of blockchain applications; standards and interfaces related to blockchain; organizational implications and legal issues. Also, [18] point to research needs regarding trust and collaboration in blockchain platform ecosystems. Research questions include how both trust and anonymity can be guaranteed in blockchain-mediated networks, how risks can be identified and mitigated, and how organizational and managerial issues in blockchain platforms such as financial structure, business models and pricing strategies can be resolved, how blockchain platforms function without central authority, how open these platforms are for contributions and participation, and which incentives are effective for participating developers. In [20], a research agenda for blockchain applications to business process management is proposed, including the development of appropriate execution and monitoring systems, the feasibility of using blockchain for process-aware information systems, the application of design science approaches combined with software engineering; and the impact on strategy and governance of blockchains.

There is also attention for legal and regulatory issues in relation to blockchain. Apart from the issues addressed by [1] and [3] in this respect, and the very useful overview of legal and regulatory issues in [48], [6] studied the legal aspects of smart contracts as a specific application of blockchain. It appears that complex legal questions surround blockchain and smart contracts in the sense of liability, applicable law, jurisdiction, proper governance, dispute resolution and privacy. One of the key legal issues is the establishment of proper governance mechanisms. In this discussion, the distinction between private (permissioned) and public (permissionless) blockchains is crucial as governance models will be different.

Given the emergence of networked organizations, network governance - as opposed to governance through markets and hierarchies - has emerged as an area of interest [31]. Research in this field seems to focus mostly on understanding and explaining the reason of existence and the viability conditions of such forms. This means there is a lack of research oriented towards organizational (re-)design of networks and platforms. in particular very little is known about redesign of blockchain platforms ecosystems including governance mechanisms. Issues that can be raised are: which forms of network governance match specific characteristics of blockchain systems? How effective are current forms of governance in blockchain ecosystems? How can effective forms of 
network governance and organizational routines be designed and validated? An interesting area of research would be to investigate the applicability of collaborative network design principles and governance frameworks as described in [49] to blockchain-enabled organizational forms. In addition, we should keep an eye on the limits of the extremely decentralized organizations enabled by blockchain technologies [50] and find out what the new principles for blockchain-enable collaborative organizations are.

The issue of adoption and acceptation, and readiness (mastering a wide range of capabilities), is of prime importance. There is a clear challenge of transition towards blockchain-enabled collaborative networks based on the view of blockchain applications as complex socio-technical systems. Adopting blockchain implies engaging in a systemic transition process. Studies such as [32] and [[34] state that blockchain is a foundational technology and not just a disruptive technology. The impact might be transformational but will take decades to become clear as with the internet, and the process of adoption will not be radical but gradual and steady [34].

Research of blockchain in relation to organizational innovation and collaborative networks is in the early stages. Especially there is a need for insights in organizational change and redesign during the process of blockchain adoption and in the context of business model innovation. Design and change management approaches will be useful. Collaborative networked organizations and network governance perspectives could learn from each other and apply to blockchain. Blockchain applications must be considered in the context of socio-technical systems, and the transition towards such blockchain systems provides a challenging area of research.

\section{References}

1. Tapscott D., Tapscott A.: Realizing the Potential of Blockchain. A Multi-Stakeholder Approach to the Stewardship of Blockchain and Cryptocurrencies. White Paper, World Economic Forum (2017).

2. Kashyap, M., Shipman, J., Garfinkel, H.: PwC Global FinTech Report. Price Waterhouse Cooper (2017). Retrieved: www.pwc.com/fintechreport.

3. Tapscott, D., Tapscott A.: Blockchain Revolution. How the Technology Behind Bitcoin is Changing Money, Business and the World. Portfolio Penguin, London (2016).

4. Nakamoto, S.: Bitcoin: A Peer-to-Peer Electronic Cash System (2008). Retrieved: https://bitcoin.org/bitcoin.pdf

5. Swan, M.: Blockchain. Blueprint for a New Economy. O'Reilly Media, Sebastopol (2015).

6. Rikken, O. et al.: Smart Contracts as a Specific Application of Blockchain Technology. Dutch Blockchain Coalition (2017).

7. Prinz, W., Schulte A.T.: Blockchain und Smart Contracts. Technologien, Forschungsfragen und Anwendungen. Fraunhofer-Gesellschaft (2017).

8. Gatteschi, V., Lamberti, F., Demartini, C., Pranteda, C., Santamaría, V.: Blockchain and Smart Contracts for Insurance: Is the Technology mature Enough? Future Internet 10, 20 (2018).

9. Buterin, V.: A Next-Generation Smart Contract and Decentralized Application Platform (2014). Retrieved from: https://github.com/ethereum/wiki/wiki/White-Paper.

10. Deloitte: Taking Blockchain Live. The 20 Questions that Must be Answered to Move Beyond Proofs of Concept. Deloitte Development LLC (2017). 
11. Deventer, O., Brewster, C., Everts, M.: Governance and Business Models of Blockchain Technologies and Networks. TNO White Paper, June (2017).

12. Buterin, V.: On Public and Private Blockchains. Ethereum Blog, August $7^{\text {th }}$ (2015). Retrieved: https://blog.ethereum.org/2015/08/07/on-public-and-private-blockchains/

13. Macedo, P., Camarinha-Matos, L.: Value Systems Alignment Analysis in Collaborative Networked Organizations Management. Applied Sciences, 7, 1231 (2017). Retrieved: www.mdpi.com/2076-3417/7/12/1231/pdf.

14. Böhme, R., Christin, N., Edelman, B., Moore, T.: Bitcoin: Economics, Technology, and Governance. Journal of Economic Perspective, 29(2), Spring, 213-238 (2015).

15. De Filippi, P., Loveluck B.: The Invisible Politics of Bitcoin: Governance Crisis of a Decentralized Infrastructure. Internet Policy Review 5, 3 (2016).

16. Van Alstyne, M.: Why Bitcoin has Value. Communications of the ACM 57(5), 30-32 (2014).

17. De Filippi, P.: What Blockchain Means for the Economy. Harvard Business Review, March 15 (2017)

18. Lindman, J., Rossi, M., Tuunainen, V.K.: Opportunities and Risks of Blockchain Technologies in Payments - A Research Agenda. In: Proceedings of the 50th Hawaii International Conference on System Sciences (2017).

19. Mendling, J. et al.: Blockchains for Business Process Management - Challenges and Opportunities. ACM Transactions on Management Information Systems. In press, accepted, January (2018).

20. Fridgen, G., Radszuwill, S., Urbach, N., Utz, L.: Cross-Organizational Workflow Management Using Blockchain Technology - Towards Applicability, Auditability and Automation. Conference paper, $51^{\text {st }}$ Hawaii International Conference on Systems Sciences (HICSS 2018), January (2018). Retrieved: https://www.fim-rc.de/Paperbibliothek/Veroeffentlicht/696/wi-696.pdf.

21. Psaila, S.: Blockchain: A Game Changer for Audit Processes? Deloitte Malta Article, Sept $22(2017)$

22. Grech, A., Camilleri, A.F.: Blockchain in Education. I. dos Santos, A. (ed.) EUR 28778 EN (2017).

23. Francisco, K., Swanson, D.: The Supply Chain has no Clothes: Technology Adoption of Blockchain for Supply Chain Transparency. Logistics 2(1), 2 (2018). Retrieved: http://www.mdpi.com/2305-6290/2/1/2

24. Korpela, K., Hallikas, J., Dahlberg, T.: Digital Supply Chain Transformation Toward Blockchain Integration. Proceedings of the 50th Hawaii International Conference on System Sciences, 4182-4191 (2017).

25. Huckle, S., Bhattacharya, R, White, M, Beloff, N.: Internet of Things, Blockchain and Shared Economy Applications. Procedia Computer Science, 98, 461-466 (2016).

26. Thoben, K.-D., Wiesner, S., Wuest, T.: Industry 4.0 and Smart Manufacturing. A Review of Research issues and Application Examples. Int. J. of Automation Technology, 11(1), 4 -16 (2017).

27. Dieterich, V., Ivanovic, M., Meier, T., Zäpfel, S., Utz, M., Sandner P.: Application of Blockchain Technology in the Manufacturing Industry. Working Paper, Frankfurt School Blockchain Center (2017).

28. Nowinski, W., Kozma, M.: How can Blockchain Technology Disrupt the Existing Business Models? Entrepreneurial Business and Economics Review, 5(3), 173-188 (2017).

29. Williamson, O.E.: Markets and Hierarchies. Analysis and Antitrust Implications. The Free Press, New York (1976). 
16 H. Schaffers

30. Jensen, M.C., Meckling, W.: Theory of the Firm, Managerial behaviour and Ownership Structure. Journal of Financial Economics, 3(4), 305-360 (1976).

31. Provan, K.G., Kernis, P.N.: Modes of Network Governance: Structure, Management and Effectiveness. Journal of Public Administration Research and Theory 18(2), pp 479-516 (2008).

32. Davidson, S., F. de Filippi, J. Potts, Economics of Blockchain (2016). Retrieved: SSRN: https://ssrn.com/abstract=2744751 or http://dx.doi.org/10.2139/ssrn.2744751

33. Benkler, Y.: The Wealth of Networks. How Social Production Transforms Markets and Freedom. Yale University Press (2007).

34. Iansiti, M., Lakhani, K.R.: The Truth about Blockchain. Harvard Business Review, February (2017).

35. Tapscott, D., Tapscott, A.: How Blockchain will Change Organizations. MIT Sloan Management Review, Winter (2017).

36. Camarinha-Matos, L., Afsarmanesh, H., Galeano, N., Molina, A.: Collaborative Networked Organizations - Concepts and Practice in Manufacturing Enterprises. Computers \& Industrial Engineering, 57(1), 46-60 (2009).

37. Camarinha-Matos, L., Afsarmanesh, H.: A comprehensive Modeling Framework for Collaborative Networked Organizations. J. of Intelligent Manufacturing 18, 529-542 (2007).

38. Msanjila, S.S., Afsarmanesh, H.: Modeling Trust Relationships in Collaborative Networked Organisations. Int. J. Technology Transfer and Commercialisation, 6(1) (2007).

39. Tiwana, A.: Platform Ecosystems. Aligning Architecture, Governance and Strategy. Morgan Kaufman, Waltham (2014).

40. Loss L., Schons C.H., Neves R.M., Delavy I.L., Chudzikiewicz I.S., Vogt A.M.C.: Trust Building in Collaborative Networked Organizations Supported by Communities of Practice. In: Camarinha-Matos L.M., Afsarmanesh H., Novais P., Analide C. (eds.), Establishing the Foundation of Collaborative Networks. IFIP, Vol. 243, Springer, Boston, MA (2007).

41. De Filippi, P., Wright, A.: Blockchain and the Law: The Rule of Code (2018).

42. Atzori, M.: Blockchain Technology and Decentralized Governance: Is the State still Necessary? J. of Governance and Regulation, 6(1), 46-62 (2015).

43. Murck, P.: Who Controls the Blockchain. Harvard Business Review, April 19 (2017).

44. Yermack, D.: Corporate Governance and Blockchains. Review of Finance, 7-31 (2017).

45. Sclavounis, O.: Understanding Public Blockchain Governance, November 17 (2017). Retrieved from: https://www.oii.ox.ac.uk/blog/understanding-public-blockchain-governance/

46. Pouwelse, J., De Kok, A., Fleuren, J., Hoogendoorn, P., Vliegendhart, R., De Vos, M.: Laws for Creating Trust in the Blockchain Age. European Property Law Journal 6(3), 321-356 (2018).

47. Beck, R., Avital, M., Rossi, M., Thatcher, J.B.: Blockchain Technology in Business and Information Systems Research. Business and Information Systems Engineering 59(6), 381384 (2017).

48. Maupin, J.: Mapping the Global Legal Landscape of Blockchain and Other Distributed Ledger Technologies. Centre for International Governance Innovation, CIGI Papers Nr. 149, October (2017).

49. Shuman, J., Twombly, J.: Collaborative Networks are the Organization: An innovation in Organization Design and Management. Vikalpa: The Journal for Decision Makers, 35, 1, pp 1-14 (2010).

50. McAfee, A., Brynjolfsson, E.: Machine Platform Crowd. Harnessing our Digital Future. W.W. Norton \& Company, New York (2016). 